\title{
The Analytic Continuation of the Lippmann-Schwinger Eigenfunctions, and Antiunitary Symmetries
}

\author{
Rafael DE LA MADRID
}

Department of Physics, The Ohio State University at Newark, Newark, OH 43055 USA

E-mail: rafa@mps.ohio-state.edu

Received November 07, 2008, in final form March 30, 2009; Published online April 08, 2009

doi:10.3842/SIGMA.2009.043

\begin{abstract}
We review the way to analytically continue the Lippmann-Schwinger bras and kets into the complex plane. We will see that a naive analytic continuation leads to nonsensical results in resonance theory, and we will explain how the non-obvious but correct analytical continuation is done. We will see that the physical basis for the non-obvious but correct analytic continuation lies in the invariance of the Hamiltonian under anti-unitary symmetries such as time reversal or $\mathcal{P} \mathcal{T}$.
\end{abstract}

Key words: Lippmann-Schwinger equation; resonances; Gamow states; resonant expansions; time reversal; $\mathcal{P} \mathcal{T}$ symmetry

2000 Mathematics Subject Classification: 81S99; 81U15

\section{Introduction}

The Lippmann-Schwinger equation is one of the cornerstones of quantum scattering. It was introduced by Lippmann and Schwinger in 1950 [1], and it has become standard in scattering theory $[2,3]$.

For energies belonging to the scattering spectrum of the Hamiltonian, the Lippmann-Schwinger eigenfunctions describe scattering states. In addition, one can describe resonances by analytically continuing the Lippmann-Schwinger eigenfunctions into the resonance energies. Such analytic continuation yields, up to a factor, the Gamow states.

The purpose of this paper is to explain some of the subtleties of the analytic continuation of the Lippmann-Schwinger eigenfunctions. We will see that the naive ${ }^{1}$ analytic continuation is incompatible with standard resonance expansions, and we will therefore be forced to analytically continue the Lippmann-Schwinger eigenfunctions in a somewhat counter-intuitive way. We will find out that such counter-intuitive analytic continuation is nevertheless physically sound, because it is rooted on the invariance of the Hamiltonian under anti-unitary symmetries such as time reversal or $\mathcal{P} \mathcal{T}$. The hope is that this paper will clarify both the technicalities of and the physical basis for the results of $[4,5]$.

The structure of the paper is as follows. Section 2 is devoted to recall some well-known results of scattering theory. In Section 3, we study both the naive and the correct analytic continuations of the Lippmann-Schwinger eigenfunctions. Section 4 investigates the physical soundness of such analytic continuations. In Section 5, we state our conclusions.

${ }^{\star}$ This paper is a contribution to the Proceedings of the VIIth Workshop "Quantum Physics with NonHermitian Operators" (June 29 - July 11, 2008, Benasque, Spain). The full collection is available at http://www.emis.de/journals/SIGMA/PHHQP2008.html

${ }^{1}$ We will use the adjective "naive" for lack of a better term. 
Throughout the paper, rather than working in a general setting, we shall use the example of the spherical shell potential, although, as explained in [5], the results hold for a larger class of potentials that include, in particular, potentials of finite range.

\section{Preliminaries}

Before proceeding with analytic continuations, it is convenient to briefly recall some basic results of scattering theory. For a more detailed account on the results of this section, the reader is referred to $[2,3]$.

\subsection{The Lippmann-Schwinger eigenfunctions}

Let us take a simple example, such as the spherical shell potential:

$$
V(r)= \begin{cases}0, & 0<r<a \\ V_{0}, & a<r<b \\ 0, & b<r<\infty\end{cases}
$$

For this potential, the Lippmann-Schwinger equation

$$
\left|E^{ \pm}\right\rangle=|E\rangle+\frac{1}{E-H_{0} \pm i \epsilon} V\left|E^{ \pm}\right\rangle
$$

has the following solutions in the radial, position representation for zero angular momentum:

$$
\left\langle r \mid E^{ \pm}\right\rangle \equiv \chi^{ \pm}(r ; E)=N(E) \frac{\chi(r ; E)}{\mathcal{J}_{ \pm}(E)},
$$

where $N(E)$ is a $\delta$-normalization factor,

$$
N(E)=\sqrt{\frac{1}{\pi} \frac{2 m / \hbar^{2}}{\sqrt{2 m / \hbar^{2} E}},}
$$

$\chi(r ; E)$ is the regular solution,

$$
\chi(r ; E)= \begin{cases}\sin \left(\sqrt{\frac{2 m}{\hbar^{2}} E r}\right), & 0<r<a, \\ \mathcal{J}_{1}(E) e^{i \sqrt{\frac{2 m}{\hbar^{2}}\left(E-V_{0}\right)} r}+\mathcal{J}_{2}(E) e^{-i \sqrt{\frac{2 m}{\hbar^{2}}\left(E-V_{0}\right)} r}, & a<r<b, \\ \mathcal{J}_{3}(E) e^{i \sqrt{\frac{2 m}{\hbar^{2}} E} r}+\mathcal{J}_{4}(E) e^{-i \sqrt{\frac{2 m}{\hbar^{2}} E} r}, & b<r<\infty,\end{cases}
$$

and $\mathcal{J}_{ \pm}(E)$ are the Jost functions,

$$
\mathcal{J}_{+}(E)=-2 i \mathcal{J}_{4}(E), \quad \mathcal{J}_{-}(E)=2 i \mathcal{J}_{3}(E) .
$$

In terms of the Jost functions, the $S$ matrix is given by

$$
S(E)=\frac{\mathcal{J}_{-}(E)}{\mathcal{J}_{+}(E)}
$$

The functions $\mathcal{J}_{1}(E), \ldots, \mathcal{J}_{4}(E)$ of equation (4) are determined by the conditions that $\chi(r ; E)$ vanishes at $r=0$, and that $\chi(r ; E)$ is continuous with a continuous derivative at $r=a, b$. The explicit expressions of $\mathcal{J}_{1}(E), \ldots, \mathcal{J}_{4}(E)$ can be found in [6, Appendix A]. 
To the kets $\left|E^{ \pm}\right\rangle$, there correspond the bras $\left\langle{ }^{ \pm} E\right|$, which are solutions of the following equation:

$$
\left\langle{ }^{ \pm} E\right|=\langle E|+\left\langle{ }^{ \pm} E\right| V \frac{1}{E-H_{0} \mp i \epsilon} .
$$

Solving this equation in the radial, position representation yields the "left" Lippmann-Schwinger eigenfunctions:

$$
\left\langle{ }^{ \pm} E \mid r\right\rangle=\overline{\chi^{ \pm}(r ; E)}=\chi^{\mp}(r ; E) .
$$

For the potential (1), the Green function, the $S$ matrix and the Lippmann-Schwinger eigenfunctions depend explicitly not on $E$ but on the wave number $k$,

$$
k=\sqrt{\frac{2 m}{\hbar^{2}} E}>0 .
$$

This is why it is convenient to work with $k$ rather than with $E$. In terms of $k$, the LippmannSchwinger eigenfunctions (3) read as

$$
\chi^{ \pm}(r ; E)=\sqrt{\frac{1}{\pi} \frac{2 m / \hbar^{2}}{k}} \frac{\chi(r ; k)}{\mathcal{J}_{ \pm}(k)} .
$$

These eigenfunctions are normalized to $\delta\left(E-E^{\prime}\right)$. In order to normalize them to $\delta\left(k-k^{\prime}\right)$, we define

$$
\chi^{ \pm}(r ; k) \equiv \sqrt{\frac{\hbar^{2}}{2 m} 2 k \chi^{ \pm}}(r ; E)=\sqrt{\frac{2}{\pi}} \frac{\chi(r ; k)}{\mathcal{J}_{ \pm}(k)} .
$$

It is important to note that in this equation $k$ is positive.

\subsection{The Riemann surface for the square root function}

Analytic continuations are defined for single-valued functions. When the functions are not single-valued, the Riemann surfaces provide the means to perform analytic continuations.

For the potential (1), the multiple-valuedness of the Green function, $S$ matrix and LippmannSchwinger eigenfunctions stems from the square root function through equation (8). Thus, in our example, in order to obtain analytic continuations, we need the Riemann surface for the square root function. This Riemann surface is well known, and it has been schematically displayed in Figs. 1 and 2. As shown in these figures, the positive real axis of the energy plane corresponds to the spectrum of the Hamiltonian. The upper rim of the positive $E$-axis maps onto the positive $k$-axis, and the lower rim of the positive $E$-axis maps onto the negative $k$-axis. The upper (respectively lower) half plane of the first (respectively second) sheet maps onto the first (respectively fourth) quadrant of the $k$-plane, see Fig. 1 . The upper (respectively lower) half plane of the second (respectively first) sheet maps onto the third (respectively second) quadrant of the $k$-plane, see Fig. 2.

The analytic continuation from the upper rim of the cut into the lower half plane of the second sheet, that is, the continuation from the positive $k$-axis into the fourth quadrant of the $k$-plane (see Fig. 1), is sometimes called the "from above to below" analytic continuation. Similarly, the analytic continuation from the lower rim of the cut into the upper half plane of the second sheet, that is, the continuation from the negative $k$-axis into the third quadrant of the $k$-plane (see Fig. 2), is sometimes called the "from below to above" analytic continuation. 

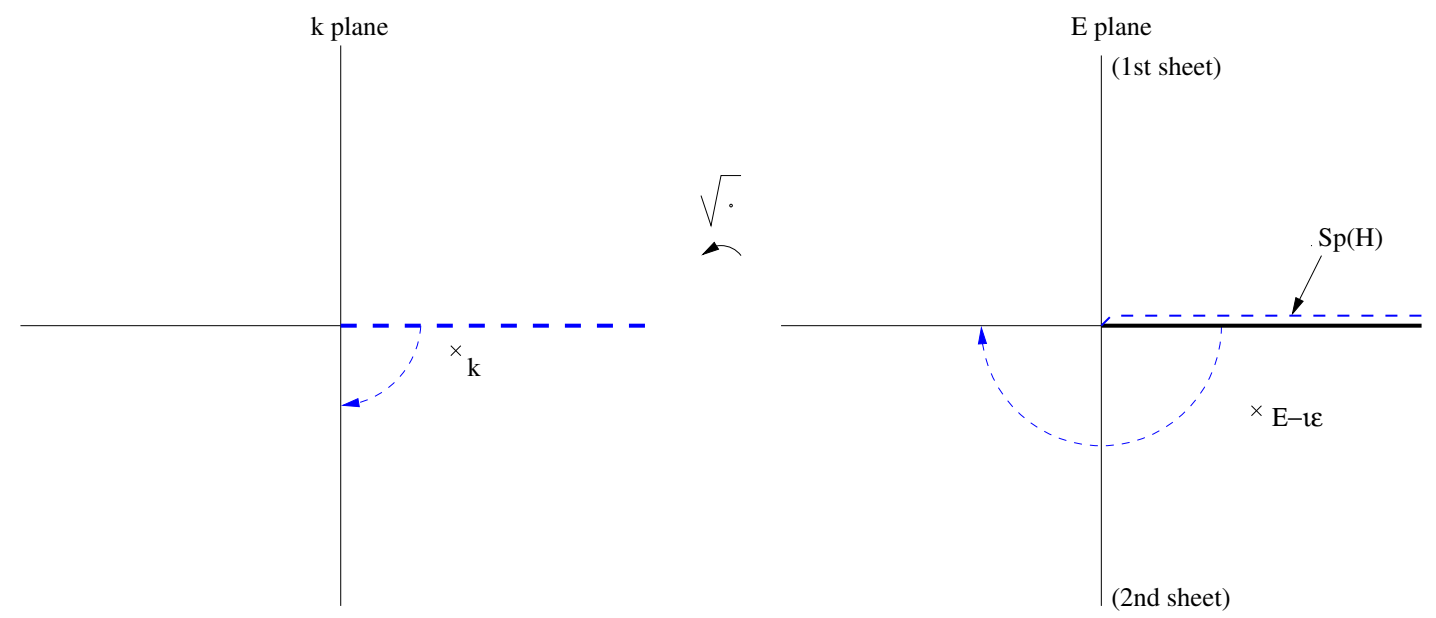

Figure 1. Analytic continuation from the upper rim of the cut into the lower half plane of the second sheet: "from above to below".
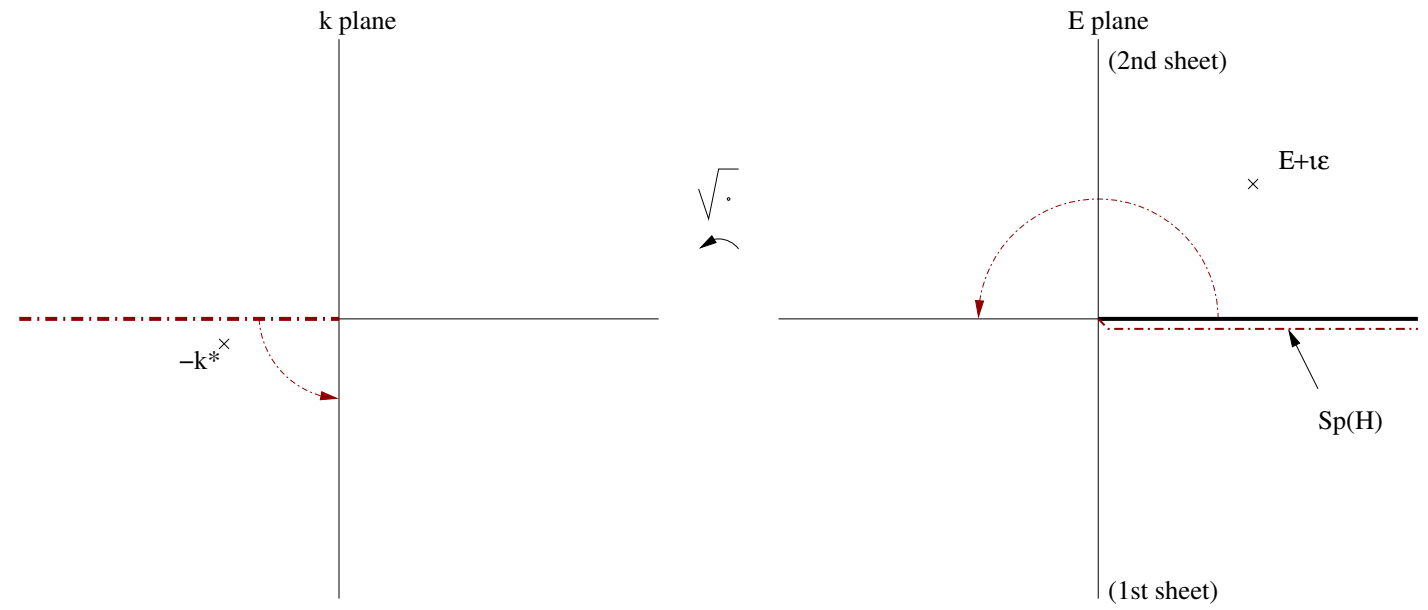

Figure 2. Analytic continuation from the lower rim of the cut into the upper half plane of the second sheet: "from below to above".

\subsection{The Gamow eigenfunctions}

The Gamow eigenfunctions are the state vectors of resonances $[7,8,9,10,11,12,13,14,15$, 16, 17, 18, 19, 20, 21, 22, 23, 24, 25, 26, 27, 28, 29, 30, 31, 32, 33, 34, 35, 36, 37, 38, 39, 40, 41]. They solve the Schrödinger equation subject to purely outgoing boundary conditions. For the spherical shell potential, the resonance energies come in complex conjugate pairs $z_{n}, z_{n}^{*}$. The energy $z_{n}=E_{n}-i \Gamma_{n} / 2$ is the resonance pole, and it belongs to the lower half plane of the second sheet of the Riemann surface. The energy $z_{n}^{*}=E_{n}+i \Gamma_{n} / 2$ is the anti-resonance pole, and it belongs to the upper half plane of the second sheet of the Riemann surface. The corresponding resonance and anti-resonance wave numbers are given by

$$
k_{n}=\sqrt{\frac{2 m}{\hbar^{2}} z_{n}}, \quad-k_{n}^{*}=\sqrt{\frac{2 m}{\hbar^{2}} z_{n}^{*}}, \quad n=1,2, \ldots,
$$

which belong, respectively, to the fourth and third quadrants of the $k$-plane. 
In terms of the wave number $k_{n}$, the $n$th Gamow eigensolution reads

$$
u\left(r ; z_{n}\right)=u\left(r ; k_{n}\right)=N_{n} \begin{cases}\frac{1}{\mathcal{J}_{3}\left(k_{n}\right)} \sin \left(k_{n} r\right), & 0<r<a, \\ \frac{\mathcal{J}_{1}\left(k_{n}\right)}{\mathcal{J}_{3}\left(k_{n}\right)} e^{i Q_{n} r}+\frac{\mathcal{J}_{2}\left(k_{n}\right)}{\mathcal{J}_{3}\left(k_{n}\right)} e^{-i Q_{n} r}, & a<r<b, \\ e^{i k_{n} r}, & b<r<\infty,\end{cases}
$$

where $N_{n}$ is a normalization factor,

$$
N_{n}^{2}=i \operatorname{res}[S(k)]_{k=k_{n}},
$$

and where

$$
Q_{n}=\sqrt{\frac{2 m}{\hbar^{2}}\left(z_{n}-V_{0}\right)}
$$

The Gamow eigensolution associated with the $n$th anti-resonance pole reads

$$
u\left(r ; z_{n}^{*}\right)=u\left(r ;-k_{n}^{*}\right)=M_{n} \begin{cases}\frac{1}{\mathcal{J}_{3}\left(-k_{n}^{*}\right)} \sin \left(-k_{n}^{*} r\right), & 0<r<a, \\ \frac{\mathcal{J}_{1}\left(-k_{n}^{*}\right)}{\mathcal{J}_{3}\left(-k_{n}^{*}\right)} e^{-i Q_{n}^{*} r}+\frac{\mathcal{J}_{2}\left(-k_{n}^{*}\right)}{\mathcal{J}_{3}\left(-k_{n}^{*}\right)} e^{i Q_{n}^{*} r}, & a<r<b, \\ e^{-i k_{n}^{*} r}, & b<r<\infty,\end{cases}
$$

where $M_{n}$ is a normalization factor,

$$
M_{n}^{2}=i \operatorname{res}[S(k)]_{k=-k_{n}^{*}}=\left(N_{n}^{2}\right)^{*},
$$

and where

$$
-Q_{n}^{*}=\sqrt{\frac{2 m}{\hbar^{2}}\left(z_{n}^{*}-V_{0}\right)} .
$$

\subsection{The analyticity structure of the "total" Green function}

The Green function is the kernel of the resolvent when expressed as an integral operator. In Dirac's bra-ket notation, it reads

$$
G(r, s ; z)=\left\langle r\left|\frac{1}{z-H}\right| s\right\rangle, \quad z \in \mathbb{C} .
$$

Since our potential does not bind bound states, the Green function is analytic in the first sheet of the Riemann surface except for the positive real axis, the cut, where it is discontinuous. Thus, when we approach the cut from above we obtain a limit $G^{+}$different from the limit $G^{-}$that we obtain when we approach the cut from below:

$$
G^{ \pm}(r, s ; E)=\lim _{\operatorname{Im}(z) \rightarrow 0} G(r, s ; z), \quad z=E \pm i \operatorname{Im}(z), \quad E, \operatorname{Im}(z)>0 .
$$

The limits $G^{ \pm}(r, s ; E)$ are the well-known retarded and advanced propagators, which in bra-ket notation are denoted as

$$
G^{ \pm}(r, s ; E)=G(r, s ; E \pm i 0)=\left\langle r\left|\frac{1}{E-H \pm i \epsilon}\right| s\right\rangle, \quad E \geq 0
$$



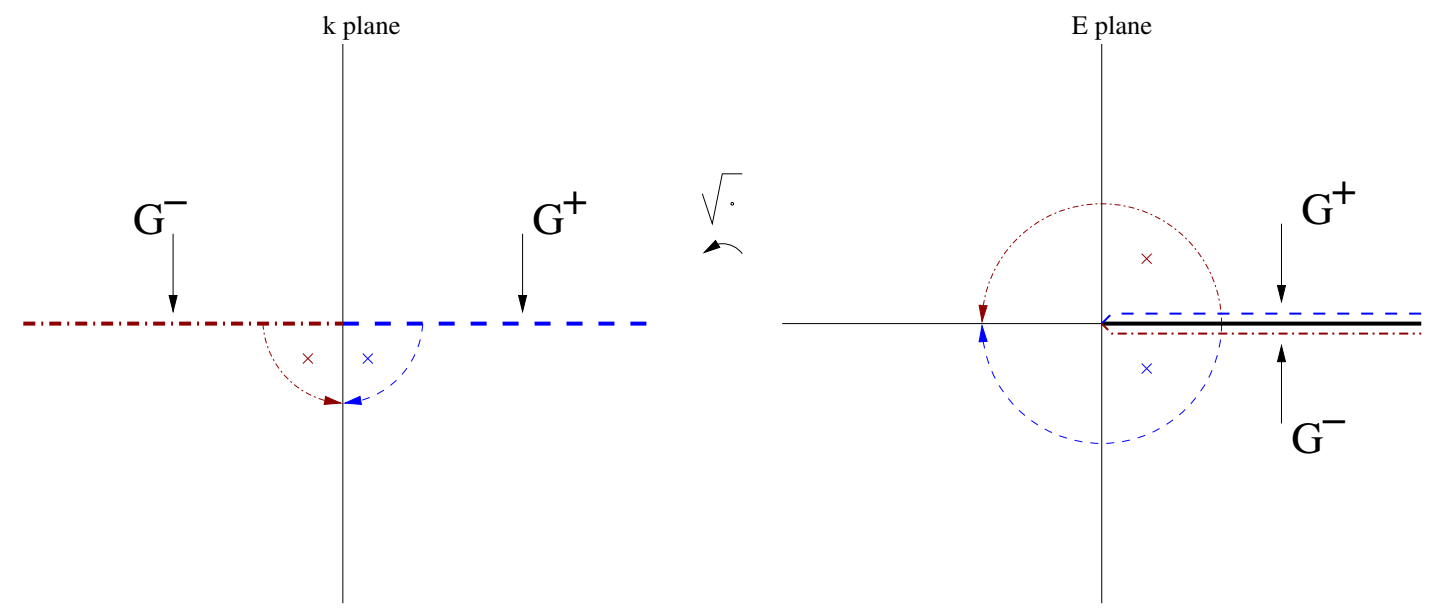

Figure 3. The "from below to above" and the "from above to below" analytic continuations of the Green function.

where $E \pm i 0 \equiv E \pm i \epsilon$ denote the energies on the upper and lower rims of the cut. These propagators are related by complex conjugation:

$$
G^{+}(r, s ; E)=\overline{G^{-}(r, s ; E)} .
$$

Now, in order to obtain a resonance energy, we continue $G^{+}(r, s ; E)$ "from above to below" till we reach a pole in the lower half plane of the second sheet, see Fig. 3. Similarly, in order to obtain an anti-resonance energy, we continue $G^{-}(r, s ; E)$ "from below to above" till we reach a pole in the upper half plane of the second sheet, see Fig. 3.

The above analytic continuations have counterparts in terms of the wave number. If we denote the complex wave number by $q$, we have that

$$
\begin{aligned}
& G^{+}(r, s ; k)=\lim _{\operatorname{Im}(q) \rightarrow 0} G(r, s ; q), \quad q=k+i \operatorname{Im}(q), \quad \operatorname{Im}(q), k>0, \\
& G^{-}(r, s ;-k)=\lim _{\operatorname{Im}(q) \rightarrow 0} G(r, s ; q), \quad q=-k+i \operatorname{Im}(q), \quad \operatorname{Im}(q), k>0 .
\end{aligned}
$$

Thus, $G^{+}\left(G^{-}\right)$is the boundary value of $G$ on the positive (negative) $k$-axis. In terms of the wave number, the "from above to below" analytic continuation entails the continuation of $G^{+}(r, s ; k)$ from the positive $k$-axis into the fourth quadrant of the complex $k$-plane, and the "from below to above" analytic continuation entails the continuation of $G^{-}(r, s ;-k)$ from the negative $k$-axis into the third quadrant of the complex $k$-plane, see Fig. 3.

\subsection{The analyticity structure of the "free" Green function}

The "free" Green function,

$$
G_{0}(r, s ; z)=\left\langle r\left|\frac{1}{z-H_{0}}\right| s\right\rangle, \quad z \in \mathbb{C},
$$

has the same analyticity structure as the "total" Green function, except that the analytic continuation of the "free" Green function does not have any poles. The boundary values of $G_{0}(r, s ; z)$ on the upper and lower rims of the cut are the so-called "free" retarded and advanced propagators,

$$
G_{0}^{ \pm}(r, s ; E)=G_{0}(r, s ; E \pm i 0)=\left\langle r\left|\frac{1}{E-H_{0} \pm i \epsilon}\right| s\right\rangle, \quad E \geq 0 .
$$




\subsection{The analyticity structure of the $S$ matrix}

The analyticity structure of the $S$ matrix (5) is analogous to those of the Green functions. As a function of the energy, $S(E)$ has a cut through the positive real line. The boundary value on the upper rim of the cut, $S(E+i 0)$, is the complex conjugate of the boundary value on the lower rim of the cut, $S(E-i 0)$ :

$$
S(E+i 0)=\overline{S(E-i 0)} .
$$

In the wave-number plane, equation (10) becomes

$$
S(k)=\overline{S(-k)}, \quad k>0 .
$$

The poles of the analytic continuation of $S(E+i 0)$ "from above to below", i.e., the poles of the analytic continuation of $S(k)$ into the fourth quadrant, yield the resonance energies. The poles of the analytic continuation of $S(E-i 0)$ "from below to above", i.e., the poles of the analytic continuation of $S(-k)$ into the third quadrant, yield the anti-resonance energies.

The analytic properties of the $S$-matrix can be obtained through very general arguments for a large class of potentials, see e.g. [42, Chapter 2]. Essentially, one starts from the values $S(E+i 0)$ that the $S$ matrix takes on the upper rim of the cut, i.e., from the values $S(k), k>0$, that the $S$ matrix takes on the positive $k$-axis. One then continues $S(E+i 0)$ into both the first and second Riemann surfaces, i.e., one continues $S(k)$ into the whole complex $k$-plane. Such continuation has the properties mentioned above. As we shall see, the Lippmann-Schwinger bras and kets must be continued this way, too.

\section{The boundary values and the analytic continuation of the Lippmann-Schwinger bras and kets. Three resonance expansions}

After recalling the analyticity structure of the Green functions and the $S$ matrix, we are in a position to obtain the analyticity structure of the Lippmann-Schwinger eigenfunctions.

\subsection{The naive, wrong approach}

Since the boundary values $G_{0}^{ \pm}(r, s ; E)$ enter the right-hand side of the Lippmann-Schwinger equations (2) and (6), one would naively expect that the analyticity structure of the Green function carries over to the Lippmann-Schwinger bras and kets. From the Lippmann-Schwinger equations (2) and (6), from equation (9), and from the analyticity structure of the Green functions discussed in Section 2, the ket $\left|E^{+}\right\rangle$and the bra $\left\langle{ }^{-} E\right|$ seem like boundary values on the upper rim of the cut, whereas the ket $\left|E^{-}\right\rangle$and the bra $\left\langle{ }^{+} E\right|$ seem like boundary values on the lower rim of the cut, see Fig. 4 . These boundary values have counterparts in the $k$-plane. The ket $\left|E^{+}\right\rangle$and the bra $\left\langle{ }^{-} E\right|$ correspond respectively to the ket $\left|k^{+}\right\rangle$and the bra $\left\langle{ }^{-} k\right|$, which seem like boundary values on the positive $k$-axis, whereas the ket $\left|E^{-}\right\rangle$and the bra $\left\langle{ }^{+} E\right|$ correspond respectively to the ket $\left|-k^{-}\right\rangle$and the bra $\left\langle^{+}-k\right|$, which seem like boundary values on the negative $k$-axis, see Fig. 4 .

From Fig. 4, it seems clear how one should analytically continue the Lippmann-Schwinger bras and kets: The kets $\left|E^{+}\right\rangle,\left|k^{+}\right\rangle$and the bras $\left\langle^{-} E\right|,\left\langle{ }^{-} k\right|$ should be continued "from above to below," whereas the kets $\left|E^{-}\right\rangle,\left|-k^{-}\right\rangle$and the bras $\left\langle{ }^{+} E\right|,\left\langle^{+}-k\right|$ should be continued "from below to above".

However, as we are going to see through three resonance expansions, the analyticity structure of the Lippmann-Schwinger bras and kets is not that of Fig. 4. 

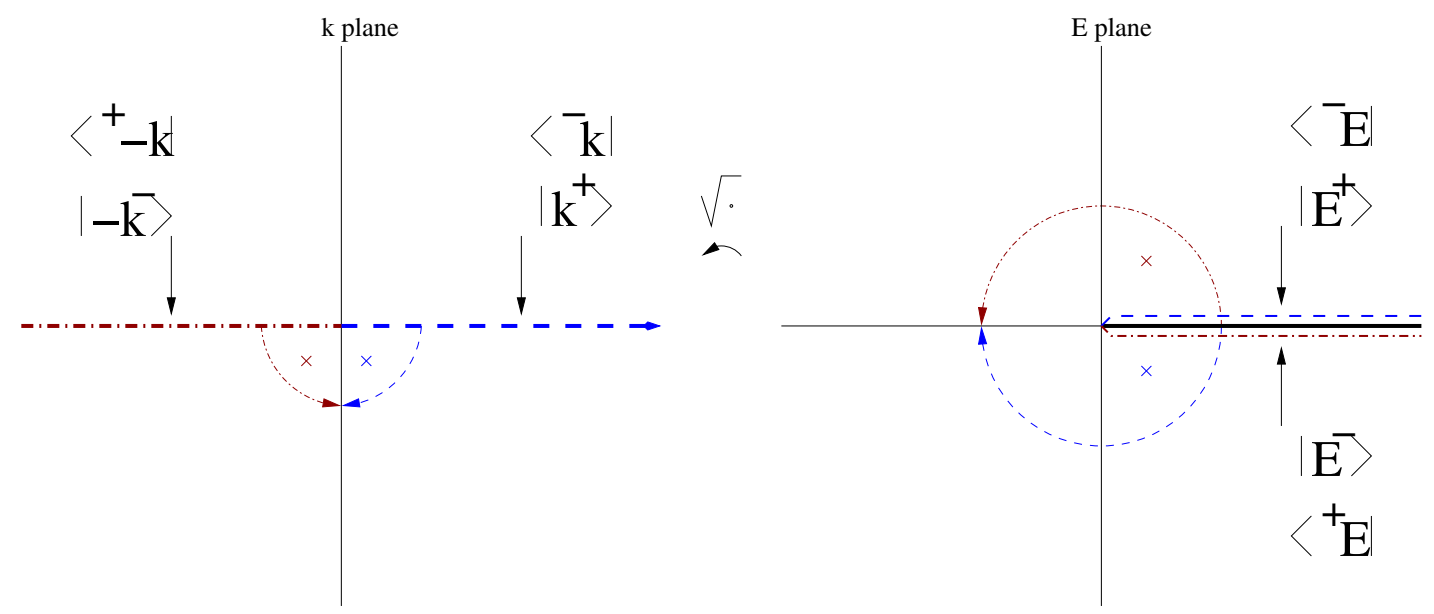

Figure 4. The naive analyticity structure of the Lippmann-Schwinger bras and kets.
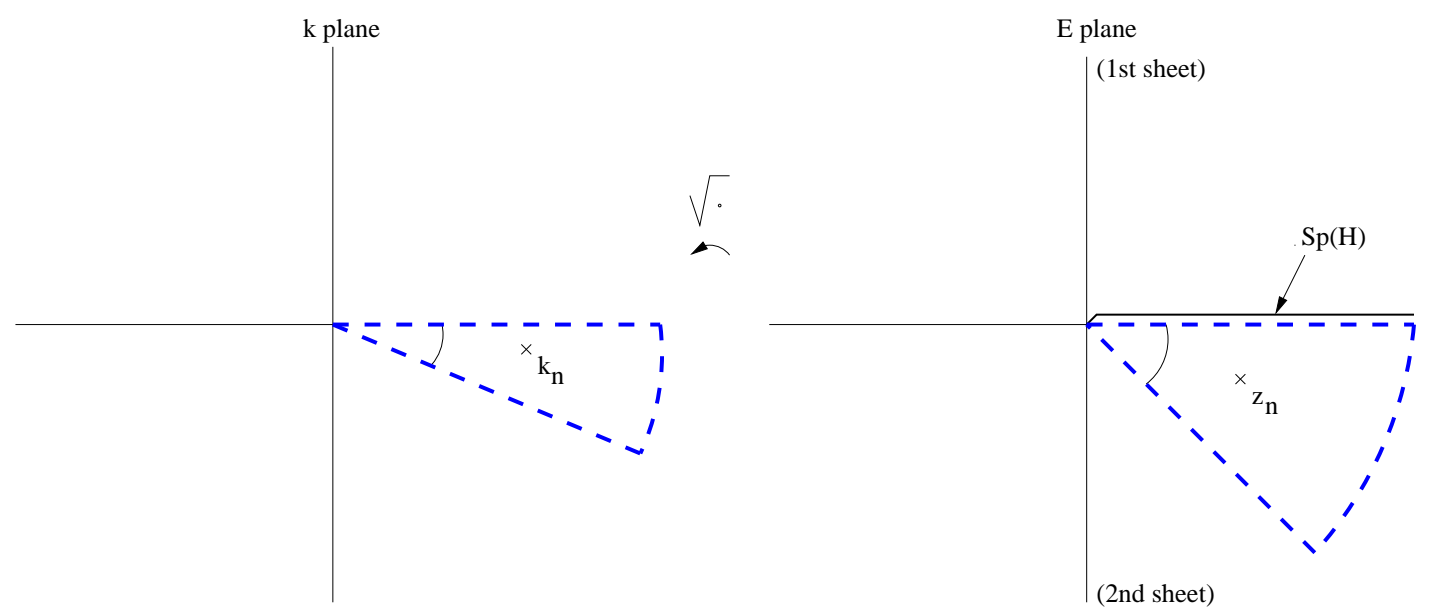

Figure 5. The contour to obtain resonance expansions. It is assumed that the contour encloses all the resonances in the lower half plane of the second sheet, and that the radius of the arc is sent to infinity.

\subsection{First resonance expansion}

The "in" bras and kets form a complete basis in the (formal) sense that

$$
1=\int_{0}^{\infty} d E\left|E^{+}\right\rangle\left\langle^{+} E\right|
$$

where 1 is the identity operator. In terms of $k$, equation (11) reads as

$$
1=\int_{0}^{\infty} d k\left|k^{+}\right\rangle{ }^{+} k \mid
$$

The expansion (11) can be analytically continued into the complex plane to obtain an expansion in terms of the Gamow states. Using the contour of Fig. 5 to continue (11), we obtain an expansion in terms of the Gamow states:

$$
1=\sum_{n=1}^{\infty}\left|z_{n}^{+}\right\rangle\left\langle{ }^{+} z_{n}\right|+\text { bg. }
$$


In terms of the wave number, equation (12) becomes

$$
1=\sum_{n=1}^{\infty}\left|k_{n}^{+}\right\rangle\left\langle{ }^{+} k_{n}\right|+\text { bg. }
$$

The sum in equation (12) runs over all the resonance energies in the lower half plane of the second sheet, and the sum in equation (13) runs over the corresponding resonance wave numbers in the fourth quadrant of the $k$-plane. The symbol "bg" stands for the background integral. The ket $\left|z_{n}^{+}\right\rangle$(respectively $\left|k_{n}^{+}\right\rangle$) arises from the analytic continuation of $\left|E^{+}\right\rangle$(respectively $\left|k^{+}\right\rangle$), and the bra $\left\langle{ }^{+} z_{n}\right|$ (respectively $\left\langle{ }^{+} k_{n}\right|$ ) arises from the analytic continuation of $\left\langle^{+} E\right|$ (respectively $\left.\left\langle{ }^{+} k\right|\right)$.

If the naive analyticity structure of Fig. 4 were correct, then in using the contour of Fig. 5 to obtain the above expansions, the ket $\left|E^{+}\right\rangle$would be continued "from above to below" whereas the bra $\left\langle{ }^{+} E\right|$ would be continued "from below to below", i.e., from the lower rim of the cut into the lower half plane of the second sheet, which makes no sense.

We note in passing that the expansion (12) must be obtained by applying a regulator of the form $e^{-i \alpha E}, \alpha \rightarrow 0^{+}$, to both sides of equation (11). ${ }^{2}$ The reason is that the expansion (12) acquires meaning when we apply it to well-behaved wave functions. Since in the energy representation such wave functions diverge exponentially, we need the regulator $e^{-i \alpha E}, \alpha \rightarrow 0^{+}$, to kill such exponential blowup. However, even if the wave functions didn't blow up at infinity, our conclusions would remain unaffected, since we would be nevertheless continuing $\left\langle^{+} E\right|$ "from below to below". Thus, in this paper we will not worry about such exponential blowup at infinity.

\subsection{Second resonance expansion}

The "out" bras and kets form another basis in terms of which we can formally express the identity operator:

$$
1=\int_{0}^{\infty} d E\left|E^{-}\right\rangle\left\langle^{-} E\right|
$$

By way of the contour of Fig. 5, the analytic continuation of (14) yields another resonance expansion:

$$
1=\sum_{n=1}^{\infty}\left|z_{n}^{-}\right\rangle\left\langle^{-} z_{n}\right|+\text { bg. }
$$

Now, if the naive analyticity structure of Fig. 4 were correct, then in using the contour of Fig. 5 to analytically continue (14), the ket $\left|E^{-}\right\rangle$would be continued "from below to below", which makes no sense.

We can also perform formal analytic continuations of the Lippmann-Schwinger bras and kets into the upper half plane of the second sheet, thereby obtaining the Gamow states associated with the anti-resonances. In doing so, and if Fig. 4 were correct, $\left\langle{ }^{+} E\right|$ and $\left|E^{-}\right\rangle$would be continued "from below to above", whereas $\left|E^{+}\right\rangle$and $\left\langle^{-} E\right|$ would be continued "from above to above", i.e., from the upper rim of the cut into the upper half plane of the second sheet, which does not make sense.

\subsection{Third resonance expansion}

From equations (3) and (5), it follows that

$$
\left\langle r \mid E^{+}\right\rangle=S(E)\left\langle r \mid E^{-}\right\rangle \text {. }
$$

\footnotetext{
${ }^{2}$ Physically, the regulator parameter $\alpha$ plays the role of time [32].
} 

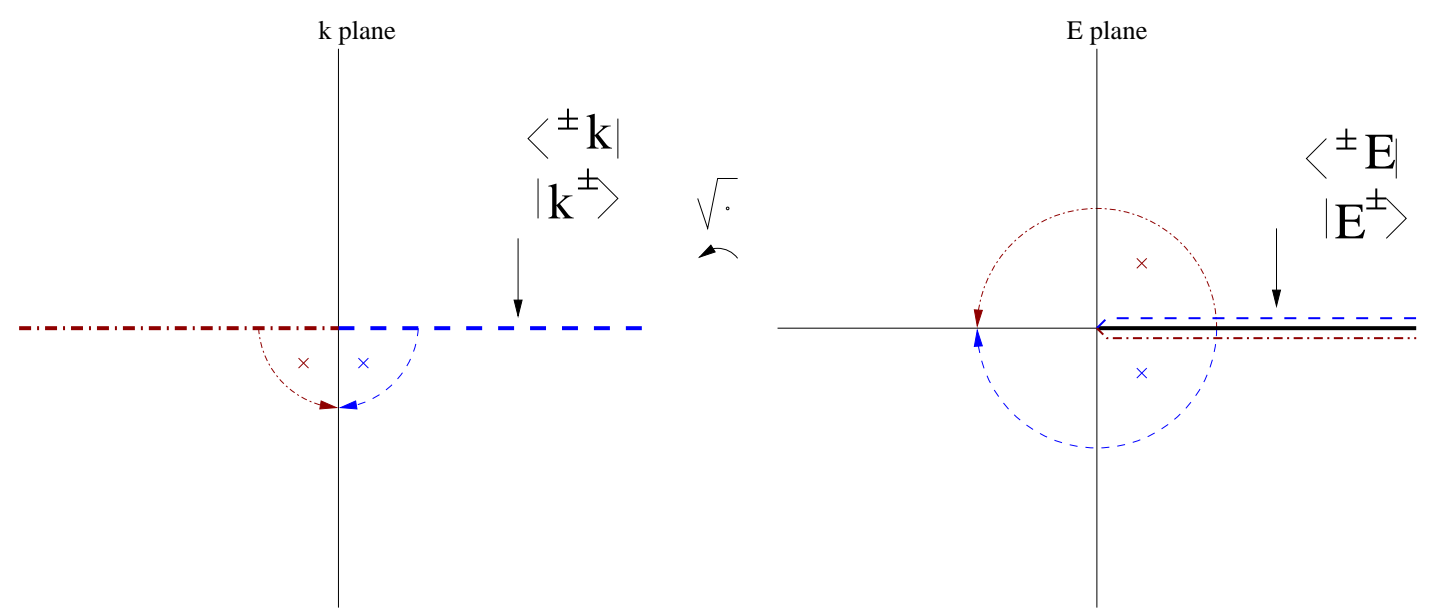

Figure 6. The Lippmann-Schwinger bras and kets as boundary values on the upper rim of the cut, from which cut they are continued into the whole complex plane.

By substituting equation (15) into equation (11), we obtain another completeness relation:

$$
1=\int_{0}^{\infty} d E\left|E^{-}\right\rangle S(E)\left\langle^{+} E\right| .
$$

Using the contour of Fig. 5, the analytic continuation of the expansion (16) yields

$$
1=\sum_{n=1}^{\infty}\left|z_{n}^{-}\right\rangle\left\langle{ }^{+} z_{n}\right|+\text { bg. }
$$

Thus, if the analyticity structure of Fig. 4 were correct, then in order to obtain (17), $\left|E^{-}\right\rangle$ and $\left\langle^{+} E\right|$ would be continued "from below to below" rather than "from above to below". Such nonsensical "from below to below" analytic continuation must be avoided.

\subsection{The Lippmann-Schwinger bras and kets as boundary values on the upper rim of the cut}

We have seen in the previous three subsections that if the Lippmann-Schwinger eigenfunctions are to be boundary values of analytic functions, and if Fig. 4 depicts those boundary values correctly, then the Lippmann-Schwinger eigenfunctions (3) and (7) are not always continued "from above to below" when we obtain resonance expansions. It is clear that the above three resonance expansions are obtained by analytically continuing the Lippmann-Schwinger bras and kets "from above to below" only if the Lippmann-Schwinger eigenfunctions (3) and (7) are all boundary values on the upper rim of the cut, that is, they are boundary values on the positive $k$-axis, see Fig. 6.

In other words, if we denote the energies on the upper rim of the cut by $E+i 0$, then the Lippmann-Schwinger eigenfunctions take the following values on that rim:

$$
\left\langle r \mid(E+i 0)^{ \pm}\right\rangle=\left\langle r \mid E^{ \pm}\right\rangle=\chi^{ \pm}(r ; E), \quad E \geq 0 .
$$

An analogous relation holds for the "left" Lippmann-Schwinger eigenfunctions:

$$
\left\langle{ }^{ \pm}(E+i 0) \mid r\right\rangle=\left\langle{ }^{ \pm} E \mid r\right\rangle=\chi^{\mp}(r ; E), \quad E \geq 0 .
$$

Equations (18) and (19) have natural counterparts in the $k$-plane. If we denote the square root of $\frac{2 m}{\hbar^{2}}(E+i 0)$ by $k, k$ being positive, then in the $k$-plane equation (18) reads as

$$
\left\langle r \mid k^{ \pm}\right\rangle=\chi^{ \pm}(r ; k), \quad k \geq 0,
$$


and equation (19) reads as

$$
\left\langle{ }^{ \pm} k \mid r\right\rangle=\chi^{\mp}(r ; k), \quad k \geq 0 .
$$

The value that the Lippmann-Schwinger eigenfunctions take on any complex energy is now obtained by analytic continuation of the eigenfunctions (18) and (19). In particular, the continuation into the resonance energies is done by continuing (18) and (19) from the upper rim of the cut into the lower half-plane of the second sheet. Likewise, the value that the LippmannSchwinger eigenfunctions take on any complex wave number is obtained by analytic continuation of the eigenfunctions (20) and (21). In particular, the continuation into the resonance wave numbers is done by continuing (20) and (21) from the positive $k$-axis into the fourth quadrant of the $k$-plane ${ }^{3}$.

Note that the analytic continuation into the anti-resonance poles, that is, the "from below to above" analytic continuation, is already determined by the values of the Lippmann-Schwinger eigenfunctions on the upper rim of the cut. One needs first to analytically continue (18) and (19) from the upper to the lower rim of cut through the first sheet of the Riemann surface. Once on the lower rim, one can continue into the anti-resonance energies. In terms of the wave number, this means we are continuing (20) and (21) from the positive to the negative $k$-axis through the upper half plane. Once on the negative $k$-axis, one can continue into the anti-resonance wave numbers.

It is worthwhile noting that the analytic continuation of the Lippmann-Schwinger eigenfunctions follows the same steps as the analytic continuation of the $S$ matrix discussed in Section 2.6. One first specifies the boundary values on the upper rim of the cut (i.e., on the positive $k$-axis), and then one obtains the values on any other complex point by analytic continuation of those boundary values.

\section{Analytic continuation, time evolution, and anti-unitary symmetries}

In the previous section, we have seen that the analytic structure implied by Fig. 4 cannot be correct, because otherwise we would perform nonsnensical analytic continuations of the LippmannSchwinger bras and kets. In this section, we are going to see that, from a physical point of view, the main reason why the analyticity structure of the Lippmann-Schwinger eigenfunctions corresponds to the one depicted in Fig. 6, rather than to the one depicted in Fig. 4, is that the time evolution of such eigenfunctions and their time reversal properties fit into Fig. 6 rather than into Fig. 4.

In order to see why, let us consider a resonant system described by a time-reversal invariant Hamiltonian $H$. Since $H$ commutes with an anti-linear operator, its complex eigenvalues must come in complex conjugate pairs ${ }^{4}$. One complex energy is the resonance energy, whereas its complex conjugate is the anti-resonance energy. Because the Gamow states satisfy purely outgoing boundary conditions only for the resonance energies $z_{n}$, whereas they satisfy purely incoming boundary conditions for the anti-resonance energies $z_{n}^{*}$, the anti-resonances are interpreted as the time-reversal of the resonances, see e.g. [44, 27]. Since the resonances (respectively, antiresonances) are obtained by analytic continuation from the upper (respectively, lower) rim of the cut into the lower (respectively, upper) half plane of the second sheet, forward time evolution must be associated with the upper rim of the cut, whereas backward (i.e., time-reversed) evolution must be associated with the lower rim of the cut.

\footnotetext{
${ }^{3}$ A different approach to analytically continue the Lippmann-Schwinger equation can be found in [43].

${ }^{4}$ As is well known, if a Hamiltonian $H$ commutes with an anti-linear operator, then $H$ has either real spectrum or its complex eigenvalues come in complex conjugate pairs.
} 
It is now clear why all the Lippmann-Schwinger eigenfunctions must be defined on the upper rim of the cut. Since the "in" eigenfunctions have initial (i.e., prepared) boundary conditions built into them, whereas the "out" eigenfunctions have final (i.e., detected) boundary conditions built into them, and since quantum scattering needs both initial and final boundary conditions, both the "in" and the "out" scattering states must appear in the time-forward evolution of a scattering system, and therefore both must be defined on the upper rim of the cut. Their boundary values on the lower rim of the cut are associated with backward time evolution. Thus, the analyticity structure of the Lippmann-Schwinger eigenfunctions must be given by Fig. 6, not by Fig. 4 .

We note that when the Hamiltonian $H$ is not invariant under time reversal but instead is invariant under an anti-unitary symmetry such as $\mathcal{P} \mathcal{T}[45,46,47,48,49,50,51,52]$, the above conclusions still hold. The only difference is that now the anti-resonance poles are the $\mathcal{P} \mathcal{T}$ reversed of the resonance poles, and that the lower rim of the cut is associated with $\mathcal{P} \mathcal{T}$-reversed time evolution.

\section{Conclusion}

A naive view of the Lippmann-Schwinger equation leads to the erroneous conclusion that its solutions have the same analyticity structure as the Green function. Such naive view leads to Fig. 4 and to the following table:

\begin{tabular}{|l|l|l|l|}
\hline Bra/Ket & Expression & Boundary value & Time evolution \\
\hline "in" ket & $\left\langle r \mid(E+i 0)^{+}\right\rangle=\chi^{+}(r ; E)$ & upper rim & forward \\
\hline "out" ket & $\left\langle r \mid(E-i 0)^{-}\right\rangle=\chi^{-}(r ; E)$ & lower rim & backward \\
\hline "in" bra & $\left\langle^{+}(E-i 0) \mid r\right\rangle=\chi^{-}(r ; E)$ & lower rim & backward \\
\hline "out" bra & $\left\langle^{-}(E+i 0) \mid r\right\rangle=\chi^{+}(r ; E)$ & upper rim & forward \\
\hline
\end{tabular}

In the naive view, $\left|E^{+}\right\rangle$and $\left\langle^{-} E\right|$ are boundary values on the upper rim of the cut, and they are used solely for time-forward evolution. For time-reversed evolution, one uses solely $\left|E^{-}\right\rangle$ and $\left\langle^{+} E\right|$, which are boundary values on the lower rim of the cut.

We have seen however that the Lippmann-Schwinger bras and kets should be viewed as boundary values on the upper rim of the cut, from which rim they can be analytically continued into the whole complex plane, much like the continuation of the $S$-matrix is done from the upper rim of the cut into the whole complex plane. All eigenfunctions are associated with forward time evolution:

\begin{tabular}{|l|l|l|l|}
\hline Bra/Ket & Expression & Boundary value & Time evolution \\
\hline "in" ket & $\left\langle r \mid(E+i 0)^{+}\right\rangle=\chi^{+}(r ; E)$ & upper rim & forward \\
\hline "out" ket & $\left\langle r \mid(E+i 0)^{-}\right\rangle=\chi^{-}(r ; E)$ & upper rim & forward \\
\hline "in" bra & $\left\langle^{+}(E+i 0) \mid r\right\rangle=\chi^{-}(r ; E)$ & upper rim & forward \\
\hline "out" bra & $\left\langle^{-}(E+i 0) \mid r\right\rangle=\chi^{+}(r ; E)$ & upper rim & forward \\
\hline
\end{tabular}

Their analytic continuation into the lower rim of the cut is then used in a time-reversed world:

\begin{tabular}{|l|l|l|l|}
\hline Bra/Ket & Expression & Boundary value & Time evolution \\
\hline "in" ket & $\left\langle r \mid(E-i 0)^{+}\right\rangle=\chi^{+}(r ; E-i 0)$ & lower rim & backward \\
\hline "out" ket & $\left\langle r \mid(E-i 0)^{-}\right\rangle=\chi^{-}(r ; E-i 0)$ & lower rim & backward \\
\hline "in" bra & $\left\langle^{+}(E-i 0) \mid r\right\rangle=\chi^{-}(r ; E-i 0)$ & lower rim & backward \\
\hline "out" bra & $\left\langle^{-}(E-i 0) \mid r\right\rangle=\chi^{+}(r ; E-i 0)$ & lower rim & backward \\
\hline
\end{tabular}




\section{Acknowledgments}

The author wishes to thank the organizers of PHHQP VII for their kind invitation to participate in the conference and for their warm hospitality.

\section{References}

[1] Lippmann B.A., Schwinger J., Variational principles for scattering processes. I, Phys. Rev. 79 (1950), 469480.

[2] Newton R.G., Scattering theory of waves and particles, 2nd ed., Springer Verlag, New York, 1982.

[3] Taylor J.R., Scattering theory, John Wiley \& Sons, Inc., New York, 1972.

[4] de la Madrid R., The rigged Hilbert space approach to the Lippmann-Schwinger equation. Part II: The analytic continuation of the Lippmann-Schwinger bras and kets, J. Phys. A: Math. Gen. 39 (2006), 39814009, quant-ph/0603177.

[5] de la Madrid R., The resonance amplitude associated with the Gamow states, Nuclear Phys. A 812 (2008), 13-27, arXiv:0810.0876.

[6] de la Madrid R., Rigged Hilbert space approach to the Schrödinger equation, J. Phys. A: Math. Gen. 35 (2002), 319-342, quant-ph/0110165.

[7] Gamow G., Zur Quantentheorie de Atomkernes, Z. Phys. 51 (1928), 204-212.

[8] Siegert A.F.J., On the derivation of the dispersion formula for nuclear reactions, Phys. Rev. 56 (1939), $750-752$.

[9] Peierls R.E., Complex eigenvalues in scattering theory, Proc. Roy. Soc. London. Ser. A 253 (1959), 16-36.

[10] Humblet J., Rosenfeld L., Theory of nuclear reactions I. Resonant states and collision matrix, Nuclear Phys. 26 (1961), 529-578.

[11] Zeldovich Ya.B., On the theory of unstable states, Sov. Phys. JETP 12 (1961), 542-545.

[12] Berggren T., On the use of resonant states in eigenfunction expansions of scattering and reaction amplitudes, Nuclear Phys. A 109 (1968), 265-287.

[13] García-Calderón G., Peierls R., Resonant states and their uses, Nuclear Phys. A 265 (1976), 443-460.

[14] Berggren T., On the interpretation of complex cross sections for production of resonant final states, Phys. Lett. B 73 (1978), 389-392.

[15] Parravicini G., Gorini V., Sudarshan E.C.G., Resonances, scattering theory, and rigged Hilbert spaces, J. Math. Phys. 21 (1980), 2208-2226.

[16] Hernández E., Mondragón A., Resonant states in momentum representation, Phys. Rev. C 29 (1984), $722-$ 738.

[17] Curutchet P., Vertse T., Liotta R.J., Resonant random phase approximation, Phys. Rev. C 39 (1989), 1020-1031.

[18] Berggren T., Lind P., Resonant state expansion of the resolvent, Phys. Rev. C 47 (1993), 768-778.

[19] Lind P., Completeness relations and resonant state expansions, Phys. Rev. C 47 (1993), 1903-1920.

[20] Berggren T., Expectation value of an operator in a resonant state, Phys. Lett. B 373 (1996), 1-4.

[21] Bollini C.G., Civitarese O., De Paoli A.L., Rocca M.C., Physical representations of Gamow states in a rigged Hilbert space, Phys. Lett. B 382 (1996), 205-208.

[22] Ferreira L.S., Maglione E., Liotta R.J., Nucleon resonances in deformed nuclei, Phys. Rev. Lett. 78 (1997), 1640-1643.

[23] Cavalcanti R.M., de Carvalho C.A.A., On the effectiveness of Gamow's method for calculating decay rates, Rev. Bras. Ens. Fis. 21 (1999), 464-468, quant-ph/9711037.

[24] de la Madrid R., Quantum mechanics in rigger Hilbert space languare, Ph.D. Thesis, Universidad de Valladolid, 2001, available at http://www.physics.ohio-state.edu/ rafa/.

[25] Id Betan R., Liotta R.J., Sandulescu N., Vertse T., Two-particle resonant states in a many-body mean field, Phys. Rev. Lett. 89 (2002), 042501, 4 pages, nucl-th/0201077.

[26] Michel N., Nazarewicz W., Ploszajczak M., Bennaceur K., Gamow shell model description of neutron-rich nuclei, Phys. Rev. Lett. 89 (2002), 042502, 4 pages, nucl-th/0201073. 
[27] de la Madrid R., Gadella M., A pedestrian introduction to Gamow vectors, Amer. J. Phys. 70 (2002), 626-638, quant-ph/0201091.

[28] Kapuscik E., Szczeszek P., Model-independent normalization condition for Gamow vectors, Czech. J. Phys. 53 (2003), 1053-1056.

[29] Hernández E., Jáuregui A., Mondragón A., Jordan blocks and Gamow-Jordan eigenfunctions associated with a degeneracy of unbound states, Phys. Rev. A 67 (2003), 022721, 15 pages, quant-ph/0204084.

[30] Michel N., Nazarewicz W., Ploszajczak M., Okolowicz J., Gamow shell model description of weakly bound nuclei and unbound nuclear states, Phys. Rev. C 67 (2003), 054311, 17 pages, nucl-th/0302060.

[31] Kapuscik E., Szczeszek P., The physical mechanism of formation of quantum mechanical Gamow states, Found. Phys. Lett. 18 (2005), 573-580.

[32] de la Madrid R., Garcia-Calderon G., Muga J.G., Resonance expansions in quantum mechanics, Czech. J. Phys. 55 (2005), 1141-1150, quant-ph/0512242.

[33] Michel N., Nazarewicz W., Ploszajczak M., Rotureau J., Antibound states and halo formation in the Gamow shell model, Phys. Rev. C 74 (2006), 054305, 6 pages, nucl-th/0609016.

[34] Rotureau J., Michel N., Nazarewicz W., Ploszajczak M., Dukelsky J., Density matrix renormalization group approach for many-body open quantum systems, Phys. Rev. Lett. 97 (2006), 110603, 4 pages, nucl-th/0603021.

[35] Michel N., Nazarewicz W., Ploszajczak M., Threshold effects in multichannel coupling and spectroscopic factors in exotic nuclei, Phys. Rev. C 75 (2007), 031301, 5 pages, nucl-th/0702021.

[36] Julve J., de Urríes F.J., Tunnelling of plane waves through a square barrier, J. Phys. A: Math. Theor. 41 (2008), 304010, 14 pages, quant-ph/0701213.

[37] Michel N., Nazarewicz W., Ploszajczak M., Continuum coupling and single-nucleon overlap integrals, Nuclear Phys. A 794 (2007), 29-46, arXiv:0707.0767.

[38] Hatano N., Sasada K., Nakamura H., Petrosky T., Some properties of the resonant state in quantum mechanics and its computation, Progr. Theor. Phys. 119 (2008), 187-222, arXiv:0705.1388.

[39] Costin O., Lebowitz J.L., Stucchio C., Ionization in a 1-dimensional dipole model, Rev. Math. Phys. 20 (2008), 835-872, math-ph/0609069.

[40] Fernandez-Garcia N., Rosas-Ortiz O., Gamow-Siegert functions and Darboux-deformed short range potentials, Ann. Physics 323 (2008), 1397-1414, arXiv:0810.5597.

[41] Michel N., Nazarewicz W., Ploszajczak M., Vertse T., Shell model in the complex energy plane, J. Phys. G: Nucl. Part. Phys. 36 (2009), 013101, 40 pages, arXiv:0810.2728.

[42] Nussenzveig H.M., Causality and dispersion relations, Mathematics in Science and Engineering, Vol. 95, Academic Press, New York - London, 1972.

[43] Bohm A., Gadella M., Dirac kets, Gamow vectors and Gel'fand triplets. The rigged Hilbert space formulation of quantum mechanics, Lecture Notes in Physics, Vol. 348, Springer-Verlag, Berlin, 1989.

[44] Sternheim M.M., Walker J.F., Non-Hermitian Hamiltonians, decaying states, and perturbation theory, Phys. Rev. C 6 (1972), 114-121.

[45] Bender C.M., Boettcher S., Real spectra in non-Hermitian Hamiltonians having PT symmetry, Phys. Rev. Lett. 80 (1998), 5243-5246, physics/9712001.

[46] Mostafazadeh A., Pseudo-Hermiticity versus $\mathcal{P} T$ symmetry: the necessary condition for the reality of the spectrum of a non-Hermitian Hamiltonian, J. Math. Phys. 43 (2002), 205-214, math-ph/0107001.

[47] Mostafazadeh A., Batal A., Physical aspects of pseudo-Hermitian and $\mathcal{P} T$-symmetric quantum mechanics, J. Phys. A: Math. Gen. 37 (2004), 11645-11679, quant-ph/0408132.

[48] Ahmed Z., Bender C.M., Berry M.V., Reflectionless potentials and PT symmetry, J. Phys. A: Math. Gen. 38 (2005), L627-L630, quant-ph/0508117.

[49] Znojil M., Coupled-channel version of the PT-symmetric square well, J. Phys. A: Math. Gen. 39 (2006), 441-455, quant-ph/0511085.

[50] Andrianov A.A., Cannata F., Sokolov A.V., Non-linear supersymmetry for non-Hermitian, non-diagonalizable Hamiltonians. I. General properties, Nuclear Phys. B 773 (2007), 107-136, math-ph/0610024.

[51] Bender C.M., Brody D.C., Jones H.F., Meister B.K., Faster than Hermitian quantum mechanics, Phys. Rev. Lett. 98 (2007), 040403, 4 pages, quant-ph/0609032.

[52] Bender C.M., Making sense of non-Hermitian Hamiltonians, Rep. Progr. Phys. 70 (2007), 947-1018, hep-th/0703096. 